\title{
Neurodevelopmental Status of Moderate to Late Preterm Infants at 6 Months Corrected Age
}

\author{
Tanjina Hoq ${ }^{1 *}$ \\ Pranab Kumar Chowdhury ${ }^{2}$ \\ Farid Uddin Ahmed ${ }^{3}$ \\ Mitra Datta ${ }^{2}$ \\ Promugdha $\mathrm{Hafiz}^{4}$ \\ Rita Das ${ }^{1}$
}

\footnotetext{
'Department of Pediatrics

Bangladesh Institute of Tropical and Infectious Diseases (BITID) Chattogram; Bangladesh.

${ }^{2}$ Department of Pediatrics Chittagong Medical College Chattogram; Bangladesh.

${ }^{3}$ Department of Community Medicine Rangamati Medical College Rangamati, Bangladesh.

${ }^{4}$ Child Development Centre Chittagong Medical College Hospital Chattogram; Bangladesh.
}

\begin{abstract}
Background: Moderate Preterm (MP) and Late Preterm (LP) infants together comprise more than $80 \%$ of all prematurely born infants. Since last decade, there has been growing concern about Neurodevelopmental Abnormality (NDA) in these infants. This study was aimed to assess neurodevelopmental status of moderate to late preterm born neonates and to determine factors associated with adverse neurodevelopmental outcome in a tertiary hospital in Bangladesh.
\end{abstract}

Materials and methods: In this prospective observational study 215 preterm (32 to $<37$ completed weeks gestation at birth) infants (107 MP and 108 LP) were enrolled from the Special Care Neonatal Unit (SCANU) Chittagong Medical College Hospital from June 2018 to May 2019. Neurodevelopmental assessment was done by Rapid Neurodevelopmental Assessment (RNDA) at 1, 3 and 6 months of Corrected Age (CA).

Results: Of the 215 enrolled children, 63 (29.3\%) attended all the 3 follow-up, 123 (57.2\%) had incomplete follow-up or lost and 29 (13.5\%) died. At final follow-up out of 63 infants 43 (68.3\%) had no abnormality in all 8 domains of RNDA. Seven infants (11.1\%) had abnormality in one domain and 13 (20.6\%) had abnormality in more than one domain. Gross motor abnormality was most common abnormality (28.6\%), followed by cognition (14.3\%), vision (12.7\%) and behavior (12.7\%). Small for gestational age and lower birth weight were found to be independent predictive factors for NDA in moderate to late preterm infants.

Conclusion: Study has shown that NDA persist till 6 months of CA in moderate to late preterm infants. It is important to closely monitor the neurodevelopmental status and provide early intervention in referred cases.

Key words: Neurodevelopmental assessment; Neurodevelopmental abnormality; Preterm.

\section{INTRODUCTION}

Preterm birth is a live birth before 37 completed weeks of gestation, with subcategories of extremely preterm $(<28$ weeks), very preterm $(<32$ weeks) and moderate to late preterm (32-37 weeks) ${ }^{1}$. Approximately 15 million babies are born preterm annually worldwide, indicating a global preterm birth rate of about $11 \%$. The burden of preterm birth is particularly high in Low- and Middle-Income Countries (LMICs) especially those in Southeast Asia and sub-Saharan Africa ${ }^{2}$. Bangladesh is one of the 10 countries with the greatest number of preterm births in the world ${ }^{3}$.

Advances in perinatal care and establishment of improved neonatal services have increased the survival rates of preterm infants all over the world including Bangladesh. However, the gains in survival have concomitantly raised the question of long-term outcomes for these infants. Premature infants are at high risk of injury, 
particularly to the immature brain, which may be critical for their development with possible adverse effects lasting into adulthood $^{4}$. Consequently, neurodevelopmental impairments, including cognitive, language, motor and neurosensory impairments as well as behavioural disorders, occur more frequently in this vulnerable group ${ }^{5}$. Despite accumulating evidence in the literature, data from Bangladesh and other LMICs are limited due to a lack of follow-up programs for high-risk infants in general and for preterm infants in particular 6 .

Information on neurological outcomes is crucial for both policy making and future planning for healthcare, social and educational services and for counseling caregivers about expected outcomes following preterm birth ${ }^{7}$. Therefore, we examined neurodevelopmental outcomes in a hospital-based cohort of moderate to late preterm infants discharged from a Neonatal Intensive Care Unit (NICU) using the Rapid Neurodevelopmental Assessment (RNDA) tool at 1, 3 and 6months Corrected Age $(\mathrm{CA})^{8}$. We also estimated the prevalence of abnormal neurological examination and attempted to assess risk factors for poor outcome.

\section{MATERIALS AND METHODS}

This prospective observational study was conducted at the SCANU at Chittagong Medical College Hospital, in Chattogram city, one of the largest tertiary centers responsible for critical care of newborns in southeastern Bangladesh. The SCANU has 30 beds and 1200 admissions annually, of which the vast majority are transferred from healthcare facilities providing obstetric care and the others are admitted from home via the emergency room. The study was approved by the Ethical Review Committee of Chittagong Medical College and written parental consent was obtained for each study participant.

All preterm newborns of 32 to $<37$ completed weeks gestation admitted into SCANU from June 2018 to May 2019 were eligible for enrolment except those with perinatal asphyxia, meningitis, culture positive sepsis, hyperbilirubinemia $>$ $20 \mathrm{mg} / \mathrm{dl}$ or requirement of exchange transfusion, major morbidities such as intraventricular hemorrhage, major congenital malformation, congenital hypothyroidism.

Demographic data were obtained at enrollment including living place, maternal factors (Age, educational level and occupation) and monthly family income.Antenatal, perinatal, neonatal and relevant postnatal information including Comorbid maternal condition during pregnancy, Neonatal condition, Parity, Gestational age, Antenatal care, Mode of delivery, Place of delivery, Birth weight, Gestational weight, Length of stay in hospital and Multiple pregnancy were recorded.

At the time of discharge parents or guardians were advised to come for follow-up at one, three and six months of $\mathrm{CA}^{9}$. Periodic reminders was sent to parents through telephonic calls to improve follow-up. Neurodevelopmental assessment was done at each follow-up by RNDA tool. RNDA tool previously was shown to have acceptable reliability and validity in Bangladeshi children ${ }^{8}$. All tests were conducted by the principal investigator at hospital in a quiet room, and the results were recorded on standard assessment forms. Standard treatment was provided to the babies and appropriately intervened in the follow-up whenever required.

Data were analyzed with the help of computer software package (SPSS, version 23). Summary statistics are presented as mean (SD) or median (IQR) for continuous variables, and in absolute counts and percentages for categorical variables. To compare the continuous data between two group Independent sample $t$ test or Mann-Whitney $U$ test and among three groups ANOVA test or Kruskal Wallis Test was as appropriate. Chisquare test was used for group comparisons of categorical variables. Risk factors for adverse neurodevelopmental outcome were analyzed by logistic regression analysis. Results were shown by ORs with $95 \%$ CIs. Statistical significance was defined as $\mathrm{P} \leq 0.05$.

\section{RESULTS}

In the study 215 moderate to late preterm infants were enrolled. During the follow-up period, $13.5 \%$ (29 of 215) infants died, $37.7 \%$ (81 of 215 ) were lost to follow-up, and $48.8 \%$ (105 of 215) survived and came for at least one follow up. Of the infants who came for follow-up, 40 came only for 1 assessment, 2 came for 2 assessment and 63 came for all the three assessment. Two infants died after $1^{\text {st }}$ follow up, 2 died after $2^{\text {nd }}$ follow up.

Sociodemographic, antenatal, perinatal, neonatal and other variables were compared across those who completed Follow$\mathrm{Up}(\mathrm{F} / \mathrm{U})$ till 6 months, those who didn't complete F/U or were lost to follow-up, and those who died (Table I). Significant differences were found in mothers' vocational status (Higher proportion of mother work outside among those who completed follow-up) family income (Which was lowest among those who died), residential location (Higher proportion lived in rural area among those who died) gestational age (Was lowest among those who died), birth weight (Was lowest among those who died) RDS (higher proportion among those who died), length of hospital stay (Which was higher among those who completed follow-up) .

Table I : Characteristics of preterm infants according to their study retention

\begin{tabular}{|c|c|c|c|c|}
\hline Characteristics & $\begin{array}{r}\text { Complete F/U } \\
(\mathrm{n}=63)\end{array}$ & $\begin{array}{l}\text { Incomplete } \\
\text { F/U or Lost } \\
\quad(n=123)\end{array}$ & $\begin{array}{r}\text { Died } \\
(\mathrm{n}=29)\end{array}$ & $\mathrm{p}$ value \\
\hline Maternal age (Years) & $25(23-27)$ & $23(21-26)$ & $23(20-27)$ & $0.085^{\ddagger}$ \\
\hline Maternal education $^{\mathrm{a}}$ & $10(9-14)$ & $10(9-12)$ & $9(8-12)$ & $0.060^{\ddagger}$ \\
\hline Mother work outside & $10(15.9)$ & $5(4.1)$ & $0(0)$ & $0.005^{*}$ \\
\hline $\mathrm{MFI}^{\mathrm{b}}(1000 \mathrm{BDT})$ & $30(15-50)$ & $20(15-30)$ & $15(9-27)$ & $<0.001^{\ddagger}$ \\
\hline
\end{tabular}




$\begin{array}{lrrrr}\text { Resides in Rural area } & 34(54.0) & 86(69.9) & 24(82.8) & 0.015^{*} \\ \text { Maternal HTN/PET/Eclampsia } & 9(14.3) & 11(8.9) & 3(10.3) & 0.518^{*} \\ \text { Parity } & 2(1-2) & 2(1-3) & 1(1-3) & 0.361^{\ddagger} \\ \text { Had ANC } & 62(98.4) & 123(100.0) & 26(89.6) & 0.115^{*} \\ \text { Home delivery } & 4(6.3) & 6(4.9) & 3(10.3) & 0.510^{*} \\ \text { Delivered by CS } & 23(36.5) & 54(43.9) & 12(41.4) & 0.605^{*} \\ \text { Male infant } & 38(60.3) & 70(56.9) & 21(72.4) & 0.308^{*} \\ \text { Gestational age (Weeks) } & 33(33-35) & 34(32-35) & 32(32-34) & 0.038^{\ddagger} \\ \text { Birth weight (kg) } & 1.73 \pm 0.36 & 1.67 \pm 0.29 & 1.47 \pm 0.18 & <0.001^{\dagger} \\ \text { SGA } & 23(36.5) & 49(39.8) & 15(51.7) & 0.375^{*} \\ \text { Neonatal sepsis } & 33(52.4) & 52(42.3) & 13(44.8) & 0.423^{*} \\ \text { RDS } & 16(25.4) & 12(9.8) & 14(48.3) & 0.011^{*} \\ \text { LOS } & 14(9-20) & 10(7-14) & 12(9-16) & 0.008^{\ddagger}\end{array}$

Data are expressed either as frequency (Percentage), Median (IQR: Interquartile Range) or Mean $( \pm \mathrm{SD})$ as appropriate, $\mathrm{p}$ values were derived from *Chi-square test or ${ }^{\star}$ from ANOVA test or $\sharp$ : Kruskal Wallis Test as appropriate, a: Years of schooling, b: Monthly family income, FU: Follow-Up, ANC: Antenatal Care, CS: Cesarean Section, SGA: Small for Gestational Age, RDS: Respiratory Distress Syndrome, LOS: Length of Stay in hospital.

Out of 215 included infants only 63 had complete follow-up at 6 months of CA. Among them at 1 month of CA number of infants with abnormal domain were gross motor-18 (28.6\%) fine motor-1(1.6\%) vision-10 (15.9\%) hearing-5 (7.9\%) speech-4 (6.3\%) cognition-15 (23.8\%) and behavior- 12 (19\%). At 6 months of CA number of infants with abnormal domain were gross motor-18 (28.6\%) fine motor-3(4.8\%) vision-8 (12.7\%), hearing-3(4.8\%) speech-5 (7.9\%) cognition-9 $(14.3 \%)$ and behavior- 8 (12.7\%).

Table II : Frequency distribution of disability grade in different domains of RNDA at 1, 3 and 6 month of corrected age (CA) in moderate to late preterm infants

\begin{tabular}{|c|c|c|c|c|c|}
\hline Domain & Status & $\begin{array}{l}\text { Disability } \\
\text { grade }\end{array}$ & $\begin{array}{r}\text { Age } \\
\text { At } 1 \text { month } \\
\text { CA }\end{array}$ & $\begin{array}{r}\text { at assessme } \\
\text { At } 3 \text { month } \\
\text { CA }\end{array}$ & At 6 month \\
\hline \multirow[t]{4}{*}{ Gross motor } & Normal & & $45(71.4)$ & $41(65.1)$ & $45(71.4)$ \\
\hline & & Mild & $9(14.3)$ & $6(9.5)$ & $4(6.3)$ \\
\hline & Abnormal & Moderate & $5(7.9)$ & $8(12.7)$ & $7(11.1)$ \\
\hline & & Severe & $4(6.3)$ & $8(12.7)$ & $7(11.1)$ \\
\hline \multirow[t]{4}{*}{ Fine motor } & Normal & & $62(98.4)$ & $61(96.8)$ & $60(95.2)$ \\
\hline & & Mild & $1(1.6)$ & $2(3.2)$ & $2(3.2)$ \\
\hline & Abnormal & Moderate & $0(0)$ & $0(0)$ & $0(0)$ \\
\hline & & Severe & $0(0)$ & $0(0)$ & $1(1.6)$ \\
\hline \multirow[t]{4}{*}{ Vision } & Normal & & $53(84.1)$ & $54(85.7)$ & $55(87.3)$ \\
\hline & & Mild & $1(1.6)$ & $3(4.8)$ & $2(3.2)$ \\
\hline & Abnormal & Moderate & $6(9.5)$ & $2(3.2)$ & $1(1.6)$ \\
\hline & & Severe & $3(4.8)$ & $4(6.3)$ & $5(7.9)$ \\
\hline \multirow[t]{4}{*}{ Hearing } & Normal & & $58(92.1)$ & $58(92.1)$ & $60(95.2)$ \\
\hline & & Mild & $3(4.8)$ & $1(1.6)$ & $0(0)$ \\
\hline & Abnormal & Moderate & $1(1.6)$ & $4(6.3)$ & $2(3.2)$ \\
\hline & & Severe & $1(1.6)$ & $0(0)$ & $1(1.6)$ \\
\hline
\end{tabular}

\begin{tabular}{|c|c|c|c|c|c|}
\hline \multirow[t]{4}{*}{ Speech } & Normal & & $59(93.7)$ & $58(92.1)$ & $58(92.1)$ \\
\hline & \multirow[t]{3}{*}{ Abnormal } & Mild & $4(6.3)$ & $3(4.8)$ & $1(1.6)$ \\
\hline & & Moderate & $0(0)$ & $2(3.2)$ & $3(4.8)$ \\
\hline & & Severe & $0(0)$ & $0(0)$ & $1(1.6)$ \\
\hline \multirow[t]{4}{*}{ Cognition } & \multirow[t]{2}{*}{ Normal } & & $48(76.2)$ & $50(79.4)$ & $54(85.7)$ \\
\hline & & Mild & $6(9.5)$ & $6(9.5)$ & $2(3.2)$ \\
\hline & \multirow[t]{2}{*}{ Abnormal } & Moderate & $7(11.1)$ & $3(4.8)$ & $1(1.6)$ \\
\hline & & Severe & $2(3.2)$ & $4(6.3)$ & $6(9.5)$ \\
\hline \multirow[t]{4}{*}{ Behavior } & \multirow[t]{2}{*}{ Normal } & & $51(81.0)$ & $53(84.1)$ & $55(87.3)$ \\
\hline & & Mild & $6(9.5)$ & $4(6.3)$ & $2(3.2)$ \\
\hline & \multirow[t]{2}{*}{ Abnormal } & Moderate & $4(6.3)$ & $2(3.2)$ & $1(1.6)$ \\
\hline & & Severe & $2(3.2)$ & $4(6.3)$ & $5(7.9)$ \\
\hline Seizure & Normal & & $63(100.0)$ & $63(100.0)$ & $63(100.0)$ \\
\hline
\end{tabular}

Data were expressed as frequency (Percentage).

At final follow-up (6 months of CA) out of 63 infants 43 (68.3\%) had no abnormality in all 8 domains of RNDA tool. Seven infants (11.1\%) had abnormality in one domain and 13 $(20.6 \%)$ had abnormality in more than one domain (Figure 1).

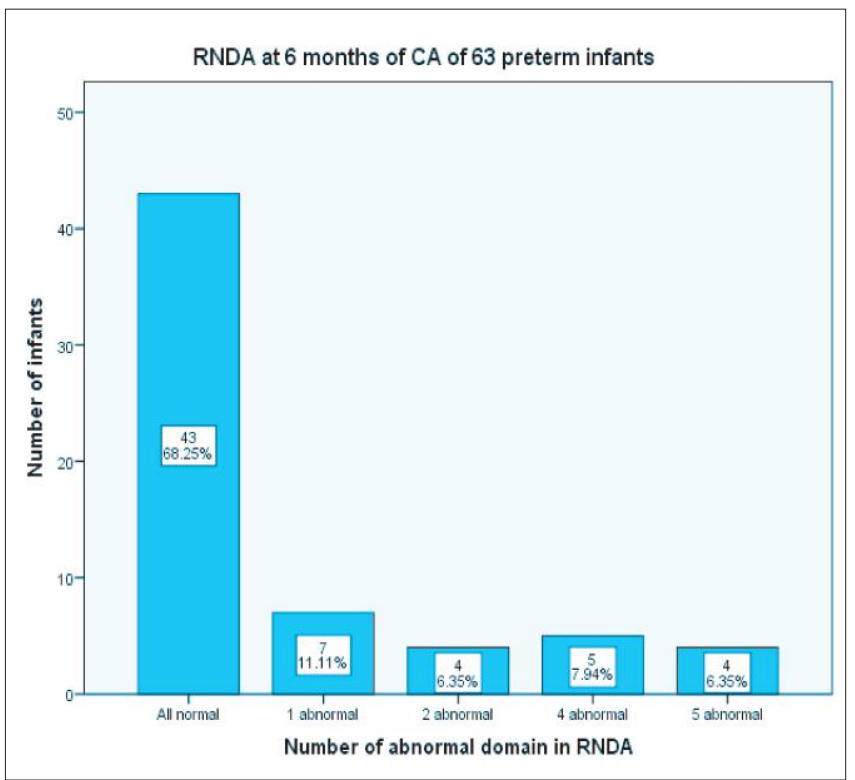

Figure 1: Clustering of abnormal domain in RNDA at 6 months of CA $(n=63)$

Association of developmental disability with sociodemographic, antenatal, perinatal, neonatal and other relevant characteristics are presented in Table III. Mode of delivery, birth weight, SGA and not exclusively breast fed had significant association with developmental disability at 6 months CA. Significantly higher number of infants with developmental disability had history of SGA $(p=0.001)$, less birth weight $(\mathrm{p}=0.016)$ and not exclusively breast fed $(\mathrm{p}=$ 0.049 ) compared to the infant with normal development. And significantly higher number of infants with normal development was delivery by CS $(\mathrm{p}=0.016)$. 
Table III : Association between socio-demographic, antenatal, perinatal, neonatal and other characteristics with developmental disability at 6 months corrected age (CA) of the 63 infants

\begin{tabular}{lrrr} 
Characteristics(unit) & $\begin{array}{r}\text { RNDA assessment at 6 month CA } \\
\text { Normal } \\
(\mathbf{n}=43)\end{array}$ & $\begin{array}{r}\text { Abnormal } \\
(\mathbf{n}=20)\end{array}$ & \\
& $24(23-27)$ & $25(22-27)$ & $0.783^{*}$ \\
Maternal age (Years) & $10(8-14)$ & $12(9-14)$ & $0.381^{*}$ \\
Maternal education ${ }^{\mathrm{a}}$ & $6(14.0 \%)$ & $4(20.0 \%)$ & $0.541^{*}$ \\
Mother work outside & $25(15-50)$ & $30(20-50)$ & $0.328^{*}$ \\
MFI $^{\text {b }}$ (1000 BDT) & $24(55.8 \%)$ & $10(50.0 \%)$ & $0.633^{*}$ \\
Resides in rural area & $9(20.9 \%)$ & $7(35.0 \%)$ & $0.350^{*}$ \\
Maternal HTN/PET/Eclampsia & $43(100 \%)$ & $19(95.0 \%)$ & $0.139^{*}$ \\
Had ANC & $1(2.3 \%)$ & $3(15.0 \%)$ & $0.055^{*}$ \\
Home delivery & $20(46.5 \%)$ & $3(15.0 \%)$ & $0.016^{*}$ \\
Delivery by CS & $27(62.8 \%)$ & $11(55.0 \%)$ & $0.566^{*}$ \\
Male infant & $34(32-35)$ & $33(33-34)$ & $0.140^{*}$ \\
Gestational age (Weeks) & $1.81( \pm 0.38)$ & $1.57( \pm 0.27)$ & $0.016^{\dagger}$ \\
Birth weight (kg) & $10(23.3 \%)$ & $13(65.0 \%)$ & $0.001^{*}$ \\
SGA & $23(53.5 \%)$ & $10(50.0 \%)$ & $0.795^{*}$ \\
Neonatal sepsis & $9(20.9 \%)$ & $7(35.0 \%)$ & $0.350^{*}$ \\
RDS & $14(8-22)$ & $14(11-15)$ & $0.882^{*}$ \\
LOS (days) & $9(20.9 \%)$ & $9(45.0 \%)$ & $0.049^{*}$ \\
Not exclusively breast fed & & & \\
\hline
\end{tabular}

Data are expressed either as frequency (Percentage) Median (IQR: Interquartile Range) or Mean $( \pm \mathrm{SD})$ as appropriate, $\mathrm{p}$ values are derived from ${ }^{*}$ Chi-square test or $^{\dagger}$ from Independent sample $t$ test or $\ddagger$ : Mann-Whiteny $U$ test as appropriate, ${ }^{\mathrm{a}}$ : Years of schooling, ${ }^{b}$ : Monthly family income, ANC: Antenatal Care, CS: Cesarean section, SGA: Small for Gestational Age, RDS: Respiratory Distress Syndrome, LOS: Length of Stay in hospital.

To determine the independent effect on NDA, the variables which had $\mathrm{p}$ value of $<0.1$ were subsequently entered into a logistic regression model. Small for gestational age and birth weight were found to be as independent factor. Infant having SGA were 11.76 times more likely to have NDA than the infant with Appropriate for Gestational Age (AGA) and as the birth weight increased chance of NDA were decreased $(p=0.016)$.

Table IV : Independent predictor of developmental abnormality by RNDA in 63 infants who came for follow up at 6 months corrected age

\begin{tabular}{lrrrr} 
Variables (unit) & Odds ratio & \multicolumn{2}{c}{$\mathbf{( 9 5 \%}$ CI for OR) } & p value \\
& & Lower & Upper & \\
Delivery by CS & 0.28 & 0.04 & 1.94 & 0.102 \\
Home delivery & 3.73 & 0.291 & 47.78 & 0.312 \\
Small for gestational age & 11.76 & 1.11 & 16.22 & 0.012 \\
Birth weight, kg & 0.117 & 0.026 & 0.673 & 0.016 \\
Not exclusively breast fed & 1.76 & 0.416 & 7.47 & 0.442 \\
\hline
\end{tabular}

CI: Confidence Interval.

\section{DISCUSSION}

Our study reports neurodevelopmental outcomes for Bangladeshi moderate to late preterm infants at 6 months CA and contributes to the evidence of adverse outcomes for preterm infants in $\mathrm{LMICs}^{10}$. We found that certain number of moderate to late preterm infants had abnormal neurodevelopment in one or more of the seven domains (Gross motor, fine motor, vision, hearing, speech, cognition, behavior). All of the infants had normal development in seizure domain. Among neurodevelopmental abnormality most prevalent was in gross motor domain followed by cognition, vision and behavior.

One in 8 infants died during the course of the study and an additional 38\% was lost to follow-up. Family income was lowest among those who died and highest among those who completed follow-up and mothers' literacy levels were highest among those who completed follow-up. All these factors, that is, rural residence, family income, gestational age, birth weight have emerged as important determinants of infant and child mortality in Bangladesh and its neighboring countries ${ }^{11,12}$.

Out of 63 infants with completed 3 follow-ups till 6 months of $\mathrm{CA}$, at 1 month of CA most frequently observed abnormal domain was gross motor $(28.6 \%)$ followed by cognition $(23.8 \%)$ behavior (19\%) and vision domain (15.9\%). At 6 months of CA abnormality in gross motor, cognition, vision and behavior were observed respectively in $28.6 \%, 14.3 \%$, $12.7 \%$ and $12.7 \%$ respectively. In a previous study from Bangladesh where RNDA tool was used among preterm infants it was observed that, more than one domain affected in $34.9 \%$ infants and the single largest category of NDA was gross motor abnormality which is greater at 3 months of age than previously in the neonatal period. In contrast, other abnormal domains have come down to lower level ${ }^{13}$.

\section{LIMITATION}

The prospective design is one of the strengths of our study and the considerable size of the initial cohort. Further, the assessment tool RNDA that we used had been previously adapted and validated for Bangladeshi infants. On the other hand, we acknowledge several limitations including the bias inherent in a single-centre study. The study lacked a full-term peer control group and included only NICU graduates. These issues may limit the generalization of our findings to the broader population of children born prematurely across the country. Furthermore, nearly $38 \%$ of the infants were lost to follow-up, potentially resulting in bias. Finally, the follow-up period was short.

\section{CONCLUSION}

In conclusion, about one third moderate to late preterm infants had abnormality in one or more domain of neurodevelopment assessed at 6 months CA. Lower birth weight and small for gestational age were found to have independent association 
with abnormal neurodevelopment. This study demonstrated that a significant proportion of preterm newborn infants in this lowresource, LMICs setting are at risk for a range of neurodevelopmental abnormality, which called attention to the importance of surveillance of the neurobehavioral development of preterm children. Currently, this burden of morbidity is largely undetected and unaddressed throughout the developing world. Our study suggests consideration of long-term neonatal follow-up programmes in Bangladesh that could assist in ensuring this vulnerable group of children fulfills their potential.

\section{DISCLOSURE}

All the authors declared no competing interest.

\section{REFERENCES}

1. Factsheet on Preterm Birth. World Health Organization. 2018. Available from: www.who.int/mediacentre/factsheets/fs363/en. [Last updated on 2018 February].

2. Walani SR. Global burden of preterm birth. Int J Gynaecol Obstet. 2020;150(1):31-33.

3. Blencowe H, Cousens S, Oestergaard M, Chou D, Moller AB, Narwal R et al. National, regional and worldwide estimates of preterm birthrates in the year 2010 with time trends since 1990 for selected countries: A systematic analysis and implications. The Lancet.2012;379(9832):2162-2172.

4. Luciana M. Cognitive development in children born preterm: Implications for theories of brain plasticity following early injury. DevPsychopathol. 2003;15(4):1017-1047.

5. Vohr BR. Neurodevelopmental outcomes of extremely preterm infants. ClinPerinatol. 2014;41(1):241-255.

6. Gladstone M, Oliver C, Van den Broek N. Survival, morbidity, growth and developmental delay for babies born preterm in low and middle income countries : A systematic review of outcomes measured. PLoS One. 2015;10(3):e0120566.

7. Council on Children with Disabilities; Section on Developmental Behavioral Pediatrics; Bright Futures Steering Committee; Medical Home Initiatives for Children With Special Needs Project Advisory Committee. Identifying infants and young children with developmental disorders in the medical home: an algorithm for developmental surveillance and screening. Pediatrics. 2006;118(1):405-420.

8. Khan NZ, Muslima H, Begum D, Shilpi AB, Akhter S, Bilkis K et al. Validation of rapid neurodevelopmental assessment instrument for undertwo-year-old children in Bangladesh. Pediatrics. 2010;125(4):e755-762.

9. Engle WA. American Academy of Pediatrics Committee on Fetus and Newborn HJ. Age terminology during the perinatal period. Pediatrics 2004;114:1362-1364.

10. Hee Chung E, Chou J, Brown KA. Neurodevelopmental outcomes of preterm infants: a recent literature review. TranslPediatr. 2020;9(Suppl 1):S3-S8.

11. Karmaker S, Lahiry S, Roy D, Singha B. Determinants of Infant and Child Mortality in Bangladesh: Time Trends and Comparisons across South Asia. BJMS.2014;13(4):431-437.

12. Dutta UP, Gupta H, Sarkar AK. Some Determinants of Infant Mortality Rate in SAARC Countries: an Empirical Assessment through Panel Data Analysis. Child Ind Res.2020;13: 2093-2116.

13. Islam MMZ, Hossain MM, Haque SA, Khan NZ. Neurodevelopmental assessment in preterm neonates at early ages: screening of at-risk Infants for long term sequlae. 2016;4(1): 5-11. 